\title{
Partnership between epigenetic reader BRD4 and transcription factor CEBPD
}

Qingwei Wang ${ }^{1}$, Mengxue Zhang ${ }^{1}$, Go Urabe ${ }^{1,2}$, Bowen Wang ${ }^{2}$, Hatice Gulcin Ozer ${ }^{3}$, Yitao Huang ${ }^{1}$, K. Craig Kent ${ }^{2}$, and Lian-Wang Guo ${ }^{1, *}$

${ }^{1}$ Department of Surgery and Department of Physiology \& Cell Biology, College of Medicine; Davis Heart and Lung Research Institute, Wexner Medical Center. The Ohio State University, Columbus, $\mathrm{OH} 43210$, USA.

${ }^{2}$ Department of Surgery, College of Medicine; Davis Heart and Lung Research Institute, Wexner Medical Center. The Ohio State University, Columbus, OH 43210, USA.

${ }^{3}$ Department of Biomedical Informatics, College of Medicine, The Ohio State University, Columbus, $\mathrm{OH} 43210$, USA.

Short title: CEBPD regulates own transcription by coupling with BRD4

* Corresponding author:

Lian-Wang Guo, Ph.D.

Department of Surgery and Department of Physiology \& Cell Biology

The Ohio State University, 473 West $12^{\text {th }}$ Ave, Columbus, OH 43210, USA

Tel: +1 614292 5276. Email: lianwang.guo@osumc.edu

Competing Interests: The authors have declared that no competing interests exist. 


\section{Abstract}

Vascular smooth muscle cell (SMC) state/phenotype transitions underlie neointimal hyperplasia $(\mathrm{IH})$ predisposing to cardiovascular diseases. Bromodomain protein BRD4 is a histone acetylation reader and enhancer mark that co-activates transcription elongation. CCAAT enhancer binding protein delta (CEBPD) is a transcription factor typically studied in adipogenesis and immune cell differentiation. Here we investigated the association between BRD4 and CEBPD in SMC state transition.

Chromatin immunoprecipitation sequencing (ChIPseq) showed enrichment of BRD4 and histone acetylation (H3K27ac) at Cebpd and enhancer in rat carotid arteries undergoing $\mathrm{IH}$. In vitro, BRD4 silencing with siRNA reduced SMC expression of CEBPD. Bromodomain-1 but not bromodoamin-2 accounted for this BRD4 function. Endogenous BRD4 co-IP'ed with CEBPD; Cebpd promoter and enhancer DNA fragments co-IP'ed with CEBPD or endogenous BRD4 (ChIP-qPCR). These co-IPs were abolished by the BRD4 bromodomain blocker JQ1. TNF $\alpha$ upregulated both BRD4 and CEBPD. Silencing CEBPD averted TNF $\alpha$-induced inflammatory SMC state transition (heightened IL-1 $\beta$, IL6, and MCP-1 mRNA levels), so did JQ1. CEBPD overexpression increased PDGFR $\alpha$ preferentially over PDGFR $\beta$; so did TNF $\alpha$, and JQ1 abolished TNF $\alpha$ 's effect.

Our data reveal a BRD4/CEBPD partnership that promotes CEBPD's own transcription and inflammatory SMC state transition, thus shedding new light on epigenetic reader and transcription factor cooperative actions in SMC pathobiology.

Keywords: BRD4, CEBPD, enhancer, ChIP, vascular smooth muscle cell state transition. 


\section{Introduction}

Smooth muscle cells (SMCs) as a major component and signaling hub maintain vascular wall homeostasis. However, upon extra- and intra-cellular perturbations, they undergo various state transitions, losing innate identity and function while acquiring new phenotypes. This SMC plasticity is now known as enabled by epigenetics ${ }^{1}$ - regulations critical in biology particularly in development and disease. Depending on micro-environmental cues, SMCs may transition to inflammatory and/or migro-proliferative or other states, contributing to a range of vascular disorders including neointimal hyperplasia $(\mathrm{IH})$ that obstructs circulation ${ }^{2}$. Therefore, in the pursuit of new interventional paradigms, it is important to interpret the $\mathrm{IH}$ pathogenic mechanisms from an epigenetic perspective. However, epigenetic determinants of SMC state transitions only begin to be unveiled ${ }^{2,3}$.

Our previous reports suggested BRD4 as a determinant of SMC state transitions in vitro and $\mathrm{IH}$ in vivo ${ }^{4,5}$, motivating further research to decipher its molecular underpinning. BRD4 belongs to the family of BETs (Bromo/ExtraTerminal domain-containing proteins), including BRD2, BRD3, BRD4, and BRDT (testis-restricted). Rapid progress, particularly in cancer research, reveals that histone acetylation reader BRD4 is closely associated with cell state/identity changes and hence critical to an array of pathogenic processes ${ }^{6}$. As a result, BRD4 is being intensively targeted in human trials, for cancers and beyond. BRD4 is found to potently prompt RNA polymerase II pause release thereby co-activating transcription elongation in response to stimulation ${ }^{6}$. This action involves multiple factors such as the elongation factor complex and central machinery of transcription. It remains poorly understood how BRD4, a seeming global regulator, assumes its functional specificity in different cell types and microenvironments ${ }^{2}$. Transcription factors (TFs) and enhancers are thought to confer BRD4 some gene loci specificity in the genomic landscape ${ }^{6,7}$. However, little is known as to how BRD4 and specific TFs and enhancers govern different SMC state transitions ${ }^{8}$.

The CCAAT enhancer binding proteins (CEBPs) comprise a family of TFs highly versatile in their pathophysiological functions. They are most notably involved in adipogenesis and immune cell differentiation among other activities. CEBPA and CEBPB are best studied, whereas CEBPD is much less understood. Evidence collectively implicates CEBPD as a central player in responses to inflammatory stimuli ${ }^{9}$, as mostly reported in immune cells with a paucity of data from SMCs. CEBPD's function is highly contextual. Dichotomous or even opposing effects mediated by CEBPD have been reported, e.g. in cell proliferation and in macrophage differentiation ${ }^{9}$. Obviously, CEBPD's functional regulations reported in other cell types or signaling contexts cannot be simply extrapolated to SMCs. Interestingly, while CEBPD expression is generally low in normal conditions, it rapidly increases in response to environmental perturbations, such as arterial injury that induces $\mathrm{IH}^{10,11}$. This raises an important question as to what epigenetic factors govern CEBPD's level and functional impact on SMC state transition ${ }^{12}$.

In the current study, as we sought to identify BRD4's downstream functional mediators and CEBPD's upstream epigenetic determinants in SMCs, the two searches converged. Through chromatin immunoprecipitation sequencing (ChIPseq), we observed that angioplasty injury induced enrichment of BRD4 and H3K27ac at the CEBPD gene. Indeed, BRD4 dominated CEBPD expression in SMCs. Moreover, CEBPD co-immunoprecipitated with BRD4, with its own promoter, and with BRD4/H3K27ac-associated enhancer. The cooperativity between BRD4 and CEBPD manifested in heightened SMC expression of pro-inflammatory cytokines. On the whole, this study explored a previously unknown area, where we found a BRD4/CEBPD partnership that promoted the inflammatory SMC state transition. 


\section{Results}

\section{Angioplasty injury induces BRD4 and H3K27ac enrichment at the CEBPD gene in rat carotid arteries}

We previously found that blocking BETs' bromodomains with JQ1 effectively curbed neointima progression in an authentic model of angioplasty-induced $\mathrm{IH}$ in rat carotid arteries ${ }^{4,13}$. While BRD4 drastically increased in the angioplasty-injured artery wall, in vitro and in vivo data suggested that BRD4 is a determinant of $\mathrm{IH}^{4,5}, 13$. In an effort to further identify the mediators of BRD4's pro-IH function, we performed high-throughput ChIPseq with injured (and uninjured) carotid arteries using the same angioplasty model of $\mathrm{IH}$. Angioplasty abruptly alters the SMC micro-environment by endothelium denudation and mechanical damage of the artery wall. This exposes SMCs to a myriad of blood-borne stimulants typical of cytokines such as TNF $\alpha$ and growth factors including PDGF, which potently trigger various SMC state transitions that perpetuate $\mathrm{IH}$. Arteries were collected for ChIPseq at post-angioplasty day 7 as this is the peak time of pro-IH molecular and cellular events ${ }^{14,15}$. Epigenomic marks involved in transcriptional activation, including BRD4, H3K27ac, and H3K4me $1^{16}$, were chosen for ChIP experiments, and the main body of data is available in our other report ${ }^{17}$. BRD4 and H3K27ac are associated with active enhancers, and H3K4me1 can be found with active, inactive, or poised enhancers.

We noticed that the ChIPseq intensity peaks for BRD4 and H3K27ac enriched at the gene of CEBPD, a TF poorly studied for SMC state transitions and $\mathrm{IH}$. Indicating genomic co-localization of BRD4, H3K27ac, and H3K4me1, their ChIPseq peaks aligned in the same genomic region (Figure 1A). Moreover, Venn diagram (Figure 1B) shows highly overlapping genes annotated based on the genomic DNA fragments that co-IP'ed with BRD4 and H3K27ac. By contrast, ChIPseq signal for H3K27me3 (a gene repression mark) was negligible. Together, these data suggest that BRD4 may regulate the expression of transcription factor CEBPD in balloon-injured rat carotid arteries.

\section{BRD4 but not BRD2 silencing diminishes CEBPD expression, so does BRD4 dominant- negative bromodomain-1}

To distinguish whether BRD4 is the only BET family member that determines CEBPD expression, genetic silencing was achieved in MOVAS (an established cell line of SMCs) using siRNAs specific to each of the 3 BETs. As shown in Figure 2A, while each siRNA was effective in silencing its respective target BET, BRD4 silencing, but not BRD2 or BRD3 silencing, led to pronounced reduction of CEBPD mRNA. This result was confirmed by Western blots, where only BRD4 silencing reduced CEBPD protein (Figure 2, B and C). BRD4 interacts with specific chromatin loci by reading/binding histone acetyl-lysine bookmarks through its two tandem bromodomains (BD1 and BD2), which are therefore crucial for BRD4's epigenetic functions. We thus dissected their role in regulating CEBPD expression. Interestingly, expressing a dominantnegative BD1 domain (i.e. a competitor to endogenous BD1) substantially reduced CEBPD protein, whereas a dominant-negative BD2 domain that proved functional in our other report ${ }^{5}$ did not have an effect herein (Figure 3, A-C). Consistently, treating SMCs with Olinone, an inhibitor selective to BD1 in BETs ${ }^{19}$, diminished CEBPD protein levels, whereas RVX208 (selective to BD2 in BETs) ${ }^{20}$ did not significantly alter CEBPD expression (Figure 3, D-F). Therefore, our data distinguished that BRD4 but not BRD2 or BRD3 was the determinant BET that controlled CEBPD expression. Furthermore, BD1 rather than BD2 was responsible for this BRD4 function; this result was unexpected given that in previous reports BD2 vs BD1 was shown to play a major role in non-SMC settings ${ }^{5,21}$. 


\section{SMC endogenous BRD4 co-immunoprecipitates with the CEBPD protein}

Reports have highlighted the importance of TFs in mediating BRD4's function of co-activating specific gene expression in cell types other than SMCs. CEBPD is a TF reportedly involved in SMC dysfunction ${ }^{22,23}$. However, a BRD4/CEBPD cooperativity in SMC pathobiology was yet to be discovered. We therefore performed co-immunoprecipitation (IP) experiments to probe a possible BRD4/CEBPD interaction. As shown in Figure 4, endogenous BRD4 IP'ed with CEBPD specifically (vs background control), suggesting their direct interaction, or indirect association but in the same protein complex. Indicative of a role for bromodomains in this interaction, the pan-BETs inhibitor JQ1 which competes with histone acetyl-lysine for binding to BD1 and BD2, blocked the BRD4/CEBPD co-IP. Furthermore, an inhibitor (TSA) of histone deacetylases commonly used to preserve histone acetylation, enhanced the co-IP of BRD4 with CEBPD. These results revealed a previously unidentified BRD4/CEBPD physical association.

\section{CEBPD and BRD4 both associate with the Cebpd promoter}

To further investigate the observed BRD4/CEBPD protein association in regulating gene expression, we performed ChIP-qPCR experiments. To validate the methodology, we chose the PDGFR $\alpha$ gene promoter for proof-of-principle since PDGFR $\alpha$ is known as a direct target of CEBPD's TF function in SMCs ${ }^{22}$. As shown in Figure 5A, treatment of SMCs with TNF $\alpha$ stimulated co-IP of PDGFR $\alpha$ promoter DNA with CEBPD. By contrast, the effect on PDGFR $\beta$ (previously regarded as non-target ${ }^{22}$ ) was minor (Figure 5B). CEBPD gain-of-function (overexpression) strongly enhanced PDGFR $\alpha$ promoter DNA pulldown (by 10-20 fold) either in the presence or absence of TNF $\alpha$, whereas no effect on PDGFR $\beta$ promoter was observed, which could serve as a negative control. Consistent with a BRD4 involvement, pretreating SMCs with JQ1 abrogated PDGFR $\alpha$ promoter pulldown that was enhanced either by TNF $\alpha$ or CEBPD overexpression.

More interestingly, software prediction suggested potential CEBPD binding sites on its own promoter. Indeed, ChIP-qPCR indicated that while the co-IP of Cebpd promoter DNA with CEBPD increased (by $\sim 2-3$ fold) due to TNF $\alpha$ stimulation, CEBPD gain-of-function further markedly enhanced this result (Figure $5 C$ ). In either case, JQ1 pretreatment abolished the enhancement of Cebpd promoter co-IP with CEBPD, suggesting an essential role of BRD4 in this interaction. BRD4 is an enhancer mark that enriched at Cebpd, and inhibiting BRD4 reduced CEBPD expression (Figures 1-3). We therefore inferred that BRD4-dependent enhancer was probably involved in the BRD4/CEBPD complex regulating Cebpd transcription. In support of this proposition, we indeed observed that BRD4-dependent enhancer DNA coIP'ed with CEBPD (Figure 5D), a result highly similar to that of Cebpd promoter in all the conditions tested here.

To further confirm an involvement of BRD4 in the transcription-regulating complex, we performed ChIP-qPCR experiments using an antibody to IP endogenous BRD4. We found that while TNF $\alpha$ stimulated the co-IP of Cebpd promoter DNA with BRD4 by $\sim 6$ fold, CEBPD overexpression further magnified this effect by $\sim 4$ fold (Figure $5 \mathrm{E}$ ). In either case, JQ1 abolished the increase of co-IP. In an effort to gain additional evidence and to expand this interesting finding beyond SMCs, we observed a similar pattern of the endogenous BRD4/Cebpd promoter co-IP using the same conditions but HEK293A cells instead of SMCs (Figure 5F).

On the whole, these (Figure 5) and other results (Figures 1-4) provided strong evidence for a multi-component complex involving $\mathrm{H} 3 \mathrm{~K} 27$ ac reader (BRD4), TF (CEBPD), enhancer enriched 
with BRD4 and H3K27ac, and Cebpd promoter DNA, which may govern CEBPD's own transcription and other target genes.

\section{CEBPD's positive role in TNF $\alpha$-induced inflammatory SMC state transition involves BRD4}

Now that the data had suggested a CEBPD (partnering with BRD4) control over its own expression, the next important question was the functional significance of this mechanism in SMC pathobiology. A role for CEBPD in proliferative SMC state transition has been reported ${ }^{22}$. However, $\mathrm{IH}$ is a complex process involving multiple SMC state transitions, a prominent one being SMC inflammation which is critically involved in $\mathrm{IH}$-associated conditions such as atherosclerosis. The inflammatory SMC state transition is typically monitored as upregulation of major pro-inflammatory cytokines including IL-1 $\beta$, IL6, and MCP-1. Both IL-1 $\beta$ and IL6 are pro$\mathrm{IH}$ and atherogenic; so is MCP-1 which is key to recruitment of inflammatory cells (e.g. activated leukocytes) to the vessel wall ${ }^{24,25}$. As indicated in Figure 6 (A-D), while the CEBPD mRNA in SMCs markedly increased after TNF $\alpha$ stimulation, this treatment also upregulated IL-1 $\beta$, IL6, and MCP-1 mRNA to a similar extent. Specifically, CEBPD gain (overexpression) and loss (silencing) of function raised and reduced the expression of these cytokines, respectively, in the absence or presence of TNF $\alpha$. CEBPD's signaling functions are highly contextual depending on cell type and stimulants ${ }^{9}$. Early evidence showed a pro-inflammatory role for CEBPD in SMCs. This observation, however, was confounded by that activation of peroxisome proliferatoractivated receptor (PPAR)-gamma, CEBPD's downstream target, inhibited CEBPD expression $^{26}$. In this regard, our study contributed an unambiguous result, that is, CEBPD plays a positive role in the specific setting of TNF $\alpha$-stimulated inflammatory SMC state transition.

It was interesting to note that TNF $\alpha$ stimulation enhanced the Cebpd promoter co-IP with BRD4 (Figure 5E) and consequently elevated CEBPD mRNA (Figure 6C). We therefore determined the effect of TNF $\alpha$ on BRD4 expression, and the data indicated that treating SMCs with TNF $\alpha$ upregulated BRD4 protein (Figure 6E). Since BRD4 rather than BRD2 or BRD3 controlled CEBPD expression via its bromodomain (Figures 2 and 3), we expected that JQ1 which blocks BET bromodomains would reveal BRD4's function in TNF $\alpha$-stimulated inflammatory SMC state transition. Indeed, pretreatment with JQ1 abrogated TNF $\alpha$-stimulated upsurge of the SMC expression of all three cytokines (IL-1 $\beta$, IL6, MCP-1) (Figure 6F). At this point, the observed BRD4/CEBPD physical association (Figures 4 and 5 ) appeared to manifest as co-function in regulating SMC inflammation, which was not previously addressed.

\section{CEBPD's preferential regulation of PDGFR $\alpha$ involves BRD4}

To further interpret the BRD4/CEBPD partnership at the next level, namely target gene expression downstream of CEBPD's TF function, we were intrigued by literature evidence of their functional convergence. While our group reported a BRD4 preferential regulation of PDGFR $\alpha$ over PDGFR $\beta$ in SMCs ${ }^{4}$, another group reported that CEBPD regulated PDGFR $\alpha$ transcription preferentially over PDGFR $\beta$ in $\mathrm{SMCs}^{22}$. It is important to address the mechanism underlying this convergence as both PDGFR $\alpha$ and PDGFR $\beta$ are potent and key signaling mediators of multiple pro-IH SMC state transitions including SMC inflammation and proliferation/migration ${ }^{4,22}$. As shown in Figure 7 (A-D), in our experiments using TNF $\alpha$ to stimulate the inflammatory SMC state transition, this treatment upregulated PDGFR $\alpha$ markedly yet PDGFR $\beta$ to a lesser degree especially at the protein level. Using JQ1 to block BRD4's function abolished this upregulation. In parallel, CEBPD gain-of-function increased PDGFR $\alpha$ mRNA by $\sim 2-3$ fold in the presence or absence of TNF $\alpha$ but insignificantly affected PDGFR $\beta$ (Figure 7, E and F). CEBPD silencing abolished TNF $\alpha$-stimulated PDGFR $\alpha$ upregulation (Figure 
7, $\mathrm{G}$ and $\mathrm{H}$ ). Of note, in accordance with the observed TNF $\alpha$-stimulated BRD4 and CEBPD upregulation (Figure 6) and their function in preferentially up-regulating PDGFR $\alpha$ vs PDGFR $\beta$ (Figure 7), TNF $\alpha$ treatment alone preferentially elevated PDGFR $\alpha$ mRA and protein levels with only a minor effect on PDGFR $\beta$ expression (Figure 7, A-H). As such, the CEBPD/BRD4 functional convergence contributes another layer of interpretation of their partnership from the angle of TNF $\alpha$-stimulated CEBPD's TF function entailing its direct target genes represented by PDGFR $\alpha$.

\section{Discussion}

IH perpetuates vascular diseases. The pathogenic basis traces to SMC state transitions, whereby SMCs acquire new phenotypes (e.g. multiplied inflammatory cytokine expression) at the expense of losing their normal homeostasis and function. This SMC identity change is increasingly recognized as propelled by epigenetic remodeling ${ }^{2}$; thus, it becomes a compelling task to interpret the largely undefined mechanisms. We tackled this issue from the perspective of an interplay between BRD4, TF, and enhancer - key factors in cell state/identity changes ${ }^{27}$. Through ChIPseq genome-wide survey using arteries undergoing $\mathrm{IH}$, we observed enrichment of BRD4 and H3K27ac at Cebpd. Genetically abating BRD4 (in vivo and in vitro) repressed Cebpd expression. Moreover, in the co-IP and ChIP-qPCR experiments, BRD4 and CEBPD were found to co-function in the same complex that also included CEBPD's own promoter. Thus, these results revealed BRD4/CEBPD physical and functional associations. This BRD4/CEBPD partnership further manifested as promoting the inflammatory SMC state transition and their preferential transcriptional control of PDGFR $\alpha$ over PDGFR $\beta$.

A unique interesting finding in this study is that CEBPD promotes its own transcription in SMCs, appearing to fit the concept of master TF. Among over a thousand TFs, a small number of socalled master TFs are critically important for cell identity, in other words, cell type or state ${ }^{7,28,29 .}$ Exemplary of master TFs, c-Myc is a powerful driver of oncogenic cell sate transitions ${ }^{7}$. In the current study, elevating CEBPD dramatically increased the expression of salient inflammatory SMC state markers including IL-1 $\beta$, IL-6, and MCP1, and silencing CEBPD kept these markers at basal levels. Obviously, CEBPD plays a key role in the transition of SMCs to an inflammatory state. Moreover, master TFs are often found to associate with enhancers and BRD4 on chromatin $^{29,30}$; so is true for CEBPD as herein shown. First, ChIPseq data obtained from chromatin samples and bioinformatics identified enhancer regions enriched with BRD4 and H3K27ac. Second, a physical association between CEBPD and BRD4 was revealed by their coIP. Third, ChIP-qPCR indicated that BRD4/H3K27ac-marked enhancer DNA was pulled down by ChIP against CEBPD. Interestingly, echoing our finding, CEBPD was recently found to bind inflammatory enhancers in aortic endothelial cells, although a BRD4 involvement was not reported $^{27}$. Another prominent feature of master TFs is their regulation of own transcription, a mechanism thought to efficiently and rapidly activate transcription and cell state transitions in response to environmental perturbation ${ }^{7}$. Indeed, ChIP-qPCR indicated that IP against CEBPD was able to pull down its own promoter DNA, indicative of a physical association.

Essentially all the CEBPD physical associations, including that with enhancer DNA, its own promoter DNA, as well as that with BRD4 (co-IP), could be abolished by using JQ1 to block BRD4's bromodomains. Importantly, aside from the CEBPD/BRD4 physical association, we also observed their functional association. First, BRD4 determined CEBPD expression levels; the 
role of BRD2 and BRD3 was minor (if any). We further distinguished that BD1 but not BD2 was critical for this BRD4 function - a finding worth more attention since BD1 and BD2 are highly similar and hence poorly differentiated in their biological functions ${ }^{5,19,21}$. Second, while TNF $\alpha$ as an extracellular stimulant upregulated CEBPD which prompted inflammatory SMC state transition (increased IL-1 $\beta$, IL6, and MCP-1), using JQ1 to block BRD4 function abolished this TNF $\alpha$ stimulation. Third, CEBPD overexpression upregulated PDGFR $\alpha$ preferentially over PDGFR $\beta$ transcription, and TNF $\alpha$ stimulation had a similar effect which was abolished by JQ1. Apparently, the BRD4 and CEBPD functions converged. Moreover, indicative of a functional hierarchy of BRD4 and CEBPD, while TNF $\alpha$ upregulated both BRD4 and CEBPD, BRD4 governed CEBPD mRNA and protein production. Although JQ1 used to block BRD4 function also binds to the bromodomains of other BETs, we could attribute its inhibitory effect to BRD4 because our data established that BRD4 but not BRD2 or BRD3 dictated CEBPD expression, as iterated above.

Thus, we have identified a novel partnership between a powerful epigenetic reader (BRD4) and a master TF (CEBPD) which are physically and functionally associated in the complex that also includes BRD4-dependent enhancer and CEBPD's own promoter. The function of this partnership manifested in the specific setting of TNF $\alpha$-induced inflammatory SMC state transition, and in the transcription of Cebpd and CEBPD's other target genes represented by PDGFR $\alpha$, a signaling mediator crucial to SMC pathophysiology. As TFs bind to sequences of promoters and enhancers that are specific to the respective genes ${ }^{27}$, a TF/BRD4 association is thought to help localize BRD4 to certain genomic loci thereby defining its functional specificity. It is noted that this TF/BRD4 pairing is highly cell type and environment/stimulation dependent ${ }^{6}$. For instance, in the inflammatory endothelial cell state transition, NF $\kappa B$ was the master TF that paired with BRD4 to potently propagate this pathogenic process ${ }^{29}$. To the best of our knowledge, the BRD4/CEBPD partnership was not previously recognized, likely due to a paucity of information on CEBPD in contrast to extensively studied CEBPA. A BRD4 functional association with CEBPA in adipogenesis was recently reported ${ }^{28,30}$, highlighting differential and contextual functions of different CEBP family members.

In the BRD4/CEBPD partnership observed here, BRD4 appears to play a central role orchestrating a multi-factor assembly that drives the inflammatory SMC state transition. As depicted in the schematic for the experimental setting herein applied, TNF $\alpha$ as extracellular signal stimulates upregulation of BRD4 protein which, while stocking up at enhancers and H3K27ac, partners with CEBPD to promote the transcription of Cebpd and other target genes, and the inflammatory SMC state transition results. This proposition is analogous to a model emerging from research in cancer and other fields ${ }^{6,7}$, namely, BRD4 "rallies" enhancer-enriched $\mathrm{TF}(\mathrm{s})$ with the transcription elongation machinery and co-factors (e.g. MED1) while reading histone acetylation marks via its bromodomain(s) ${ }^{2}$. By so doing, BRD4 may "usher" the multifactor assembly to select gene loci enabling their quick activation which drives cell state changes. As such, though seemingly a global regulator, BRD4 may assume functional specificity via context-specific associations with combinatorial TFs and enhancers at chromatin sites bookmarked (e.g. H3K27ac) in the epigenomic landscape. Nevertheless, since little is known about the BRD4/CEBPD duet in SMC (or other) cell state transitions, more research would generate exciting new knowledge to help decipher the underlying mechanisms.

\section{Conclusions}

Our results reveal a previously unidentified BRD4/CEBPD physical and functional partnership that underlies the inflammatory SMC state transition, a process permissive for $\mathrm{IH}$. A long- 
standing barrier in translational medicine is that TFs and enhancers inherently lack druggability limiting their targetability. Serendipitously, as implicated herein, their functional potency incumbent on BRD4 exposes an "Achilles' heel", that is, the druggable BRD4 bromodomains, targeting which could collapse the BRD4 assembly with context-specific TFs and enhancers. Therefore, justification is strong for more research on the BRD4/CEBPD partnership, so that essential information would become available for precision-oriented therapeutic interventions, in the vascular field or beyond.

\section{Methods}

\section{Materials}

Various resources including kits and reagents are presented in Table S1.

\section{Animals}

All animal studies conform to the Guide for the Care and Use of Laboratory Animals (National Institutes of Health) and protocols have been approved by the Institutional Animal Care and Use Committee at The Ohio State University (Columbus, Ohio). Male Sprague-Dawley rats purchased from Charles River Laboratories (Wilmington, MA) were used for experiments (at $300-350 \mathrm{~g}$ of body weight).

\section{Rat carotid artery balloon angioplasty model}

To induce $\mathrm{IH}$, angioplasty was performed to injure rat carotid arteries, following our previous reports ${ }^{4}$. Briefly, rats were kept anesthetized with $2-2.5 \%$ isoflurane (inhaling, 2 L per min). After the left common carotid artery was dissected, a 2-F balloon catheter (Edwards Lifesciences, Irvine, CA) was inserted into the common carotid artery through an arteriotomy on the external carotid artery. The balloon was inflated (at $1.5 \mathrm{~atm}$ ), withdrawn to the carotid bifurcation, and then deflated. This action was repeated three times. Perivascular BETs inhibitor administration was performed immediately following angioplasty as described below. Blood flow was finally resumed and the neck incision was closed. The animal was kept on a $37^{\circ} \mathrm{C}$ warm pad to recover. For postoperative analgesia, in addition to carprofen and bupivacaine, buprenorphine $(0.03 \mathrm{mg} / \mathrm{kg})$ was subcutaneously injected.

\section{Artery tissue ChIP sequencing and data processing}

To preserve the artery "real-time" epigenetic information, balloon-injured and uninjured (contralateral) common carotid arteries were snap frozen in liquid $\mathrm{N}_{2}$ immediately after dissected and severed out. Artery collection was performed 7 days after balloon angioplasty. Artery tissues from 40 rats were pooled for ChIPseq analysis at Active Motif per company standard procedures. Briefly, chromatin was isolated after adding lysis buffer, followed by disruption with a Dounce homogenizer. Genomic DNA was sheared to an average length of $300-500$ bp by sonicating the lysates, and the segments of interest were immunoprecipitated using an antibody $(4 \mu \mathrm{g})$ against BRD4, H3K27a, H3K27me3, or H3K4me1. The protein/DNA complexes eluted from beads were treated with RNase and proteinase K, crosslink was reversed, and the ChIP DNA was then purified for use in the preparation of Illumina sequencing libraries. Standard steps included end-polishing, dA-addition, adaptor ligation, and PCR amplification. The DNA libraries were quantified and sequenced on Illumina's NextSeq 500 , as previously described ${ }^{16}$. Sequence reads were aligned to the reference genome Rn5, peak locations were identified 
using Macs2 algorithm ${ }^{31}$ and annotated based on UCSC RefSeq. Differential peak locations were called using SICER ${ }^{32}$. In-house shell and R scripts (https://www.r-project.org) were used for data integration. To summarize and cluster genome-wide TSS coverage as heat maps, deepTools (PMID: 24799436) compute matrix and plotheatmap functions were utilized. IGV (http://www.broadinstitute.org/igv/) was used for visualization. Annotation files were downloaded from UCSC.

\section{Immunofluorescence staining on artery cross sections}

We followed our published protocol ${ }^{33}$. Briefly, artery sections were incubated without (negative staining) or with a primary antibody for $12 \mathrm{~h}$ and rinsed at least 3 times. The sections were then incubated with an anti-rabbit/mouse secondary antibody conjugated with Alexa Fluor 594 (A11037/A-21203, Invitrogen, Carlsbad, CA) and rinsed. The specific antigen was then visualized with fluorescence microscopy. Detailed information of antibodies is included in Table S2. For quantification, 5 immunostained sections from each animal were used. Fluorescence intensity in each image field was quantified by using an ImageJ software and normalized to the number of DAPI-stained nuclei in the media and neointima layers. The values from all 5 sections were pooled to generate the mean for each animal. The means from all animals in each group were then averaged, and the final mean $( \pm S E M)$ was calculated.

\section{Vascular smooth muscle cell (SMC) culture and transfection with siRNA}

SMCs (MOVAS, mouse SMC line) were cultured in DMEM supplemented with $10 \%$ FBS and $100 \mathrm{U} / \mathrm{mL}$ Penicillin-Streptomycin with $5 \% \mathrm{CO}_{2}$ at $37^{\circ} \mathrm{C}$. The construct for expressing dominantnegative BD1 of BRD4 was generated as we recently reported. For transfection with siRNA, cells cultured to $60 \% \sim 80 \%$ confluence, then RNAi Max transfection reagent was added and incubated for $12 \mathrm{~h}$. The cells recovered in fresh DMEM (no Lipofectamine, $0.5 \%$ FBS) for $12 \mathrm{~h}$ and starved for $24 \mathrm{~h}$ in DMEM with $0.5 \% \mathrm{FBS}$. The cells were then incubated with $20 \mathrm{ng} / \mathrm{ml}$ TNF $\alpha$ or solvent control $(0.1 \mathrm{BSA}, 4 \mathrm{mM} \mathrm{HCl})$ for another $24 \mathrm{~h}$ before harvest for various analyses. The siRNA sequences are listed in Table S3.

\section{Lentiviral constructs for expressing dominant-negative BRD4 bromodomains}

Construction of vectors for the expression of GFP (control) or its fusion with BRD4-BD1 or BRD4-BD2, lentivirus packaging in Lenti-X 293 cells, and transduction of MOVAS cells were performed as we recently reported ${ }^{5}$ with minor modifications. The GFP lenti-vector was kindly provided by Dr. Ming-Liang Chu (Guizhou Renmin Hospital, China). Briefly, the crude viral solution was concentrated using Lenti-x concentrator (Takara, cat.631232) to a final concentration of 108-109 IFU/ml using the Lenti-x qRT-PCR Titration Kit (Takara, cat.631235). Lentivirus with MOI of 10 was used for transduction of MOVAS cells. Cells cultured to $70 \%$ confluency were changed to starvation medium (0.5\% FBS), and lentivirus plus polybrene (Santa Cruz., Cat.sc-134220) was added and incubated for $6 \mathrm{~h}$. The cells were then cultured for recovery in fresh full medium (10\% FBS) for $24 \mathrm{~h}$ before use in experiments.

\section{SMC stable cell line for CEBPD overexpression}

The CEBPD gene was cloned into the Lenti-HA-Vector (from Addgene). For lentivirus packaging, the construct of empty vector (Lenti-HA) or that for CEBPD overexpression (LentiHA-CEBPD) transfected into the LentiX-293 cell line together with plasmids PMD2G and PSPAX2. After a $24 \mathrm{~h}$ culture, the super supernatant was collected. Lentivirus was purified and 
used to transduce the mouse smooth muscle cell line (MOVAS), and the stable cell line for the expression of HA control or HA-CEBPD was selected against puromycin.

\section{Western blot analysis}

MOVAS cells were lysed in RIPA buffer. After quantifying with DC protein assay, equal amount of protein $(20-40 \mu \mathrm{g})$ was loaded to and separated in $9 \%$ gel by SDS PAGE and then transferred to polyvinylidene difluoride (PVDF) membrane. The membrane was incubated with anti-PCNA or anti-actin overnight at $4^{\circ} \mathrm{C}$, rinsed $3 \mathrm{x}$, and then incubated with a peroxidaseconjugated secondary antibody for $1 \mathrm{~h}$ at room temperature. Specific bands were illuminated by applying enhanced chemiluminescence (ECL) reagents (Thermo Fisher Scientific; Catalog no. 32106) and then recorded with Azur LAS-4000 Mini Imager (GE Healthcare Bio-Sciences, Piscataway, New Jersey). Band intensity was quantified with ImageJ. All antibodies are included in Table S2.

\section{Quantitative real-time PCR (qRT-PCR)}

Total RNA was extracted from cell lysates using the TRIzol reagent following the manufacturer's instruction (Thermo Fisher Scientific, 15596026) and used for cDNA synthesis with the HighCapacity cDNA Reverse Transcription kit (Thermo Fisher Scientific, 4368814). In each $20 \mu \mathrm{l}$ reaction, $10 \mathrm{ng}$ of cDNA was amplified through qRT-PCR using PowerUp SYBR Green Master Mix (Thermo Fisher Scientific, A25778), and mRNA levels were determined using 7500 Fast Real-Time PCR System (Applied Biosystems, Carlsbad, CA). The data was normalized to glyceraldehyde 3-phosphate dehydrogenase (GADPH) using the $\Delta \Delta \mathrm{Ct}$ method. The primers are listed in Table S4.

\section{Co-immunoprecipitation (Co-IP)}

We used Pierce Crosslink Immunoprecipitation Kit (Thermo Scientific, 26147) by following the manufacturer's instruction. Briefly, cells were rinsed and incubated with ice-cold hypotonic buffer (2 Å $1 \mathrm{~min}, 20 \mathrm{mM}$ HEPES, $20 \%$ glycerol, $10 \mathrm{mM} \mathrm{NaCl}, 1.5 \mathrm{mM} \mathrm{MgCl} 2,0.2 \mathrm{mM}$ EDTA and $0.1 \%$ NP-40) supplemented with $1 \mathrm{mM}$ dithiothreitol, and protease and phosphatase inhibitor cocktail (Thermo Fisher Scientific, 87785). Nuclei were collected and sonicated, and the lysates were cleared by centrifugation. Magnetic beads (Dynabeads Protein A or G, Invitrogen) preloaded with an anti-HA antibody were added to the supernatant and incubated at $4{ }^{\circ} \mathrm{C}$ for $4 \mathrm{~h}$. The beads were washed $3 x$ with the binding buffer $(50 \mathrm{mM}$ Tris- $\mathrm{Cl}, 150 \mathrm{mM} \mathrm{NaCl}, 1 \mathrm{mM}$ EDTA, $10 \%$ glycerin) and SDS sample buffer was then added to elute the co-IP'ed proteins for Western blot determination.

\section{Chromatin immunoprecipitation (ChIP)-qPCR assay}

ChIP was performed as we recently reported ${ }^{34}$ using the Pierce Magnetic ChIP kit (Thermo Fisher Scientific, 26157). Briefly, cells were cross-linked with $1 \%$ formaldehyde and the reaction was stopped by glycine. The cells were washed and lysed for nuclei extraction. Micrococcal nuclease was added to the nuclei suspension to digest the DNA for $15 \mathrm{~min}$ at $37^{\circ} \mathrm{C}$, and then MNase Stop Solution was added to stop the reaction. The recovered nuclei were re-suspended in IP Dilution Buffer and sonicated (Four 5-second pulses at 20 Watts for $1 \times 10^{\wedge} 6$ cells) to disrupt the nuclear membrane. Chromatin extracts containing DNA fragments ( 500 base pairs in each) were immunoprecipitated by incubating with a specific antibody (or lgG) overnight at 
$4^{\circ} \mathrm{C}$. ChIP-grade Protein A/G Magnetic beads were added and incubated for $\sim 2-4 \mathrm{~h}$ at $4^{\circ} \mathrm{C}$. RNAse $A$ and Proteinase $K$ were used to digest RNA and protein. The purified DNA was used for qRT-PCR as described above. The primers are listed in Table S4.

\section{Statistical Analysis}

Data are presented as mean \pm standard error of the mean (SEM). Normality of the data was assessed based on Shapiro-Wilk normality test prior to statistical calculation using Prism 6.0 software (GraphPad). $p<0.05$ was considered significant. One-way ANOVA followed by Bonferroni post-hoc test was applied to, as specified in each figure legend. For ChIPseq data, statistical analyses were performed using SAS/STAT software, version 9.2 (SAS Institute, Inc., Cary, NC).

\section{Acknowledgement}

This work was supported by NIH grants R01 HL133665 (to L.-W. G.), R01HL-143469 and R01HL-129785 (to K.C.K., L.-W. G.).

\section{References}

1. Gomez D, Swiatlowska P and Owens GK. Epigenetic Control of Smooth Muscle Cell Identity and Lineage Memory. Arteriosclerosis, thrombosis, and vascular biology. 2015;35:2508-16.

2. Borck PG, Guo L.-W., Plutzky J. BET epigenetic reader proteins in cardiovascular transcriptional programs. Circulation research. 2020;126:00-00.:DOI: 10.1161/CIRCRESAHA.120.315929.

3. Stratton MS, Farina FM and Elia L. Epigenetics and vascular diseases. Journal of molecular and cellular cardiology. 2019;133:148-163.

4. Wang B, Zhang M, Takayama T, Shi X, Roenneburg DA, Kent KC and Guo LW. BET Bromodomain Blockade Mitigates Intimal Hyperplasia in Rat Carotid Arteries. EBioMedicine. 2015;2:1650-61.

5. Zhang M, Wang B, Urabe G, Huang Y, Plutzky J, Kent KC and Guo LW. The BD2 domain of BRD4 is a determinant in EndoMT and vein graft neointima formation. Cellular signalling. 2019;61:20-29.

6. Shi J and Vakoc CR. The mechanisms behind the therapeutic activity of BET bromodomain inhibition. Molecular cell. 2014;54:728-36.

7. Bradner JE, Hnisz D and Young RA. Transcriptional Addiction in Cancer. Cell. 2017;168:629-643.

8. Das S, Senapati P, Chen Z, Reddy MA, Ganguly R, Lanting L, Mandi V, Bansal A, Leung A, Zhang S, Jia Y, Wu X, Schones DE and Natarajan R. Regulation of angiotensin II actions by enhancers and super-enhancers in vascular smooth muscle cells. Nature communications. 2017;8:1467.

9. Ko CY, Chang WC and Wang JM. Biological roles of CCAAT/Enhancer-binding protein delta during inflammation. J Biomed Sci. 2015;22:6.

10. Kelkenberg U, Wagner AH, Sarhaddar J, Hecker $M$ and von der Leyen HE. CCAAT/enhancer-binding protein decoy oligodeoxynucleotide inhibition of macrophage- 
rich vascular lesion formation in hypercholesterolemic rabbits. Arteriosclerosis, thrombosis, and vascular biology. 2002;22:949-54.

11. Kaplan-Albuquerque N, Bogaert YE, Van Putten V, Weiser-Evans MC and Nemenoff RA. Patterns of gene expression differentially regulated by platelet-derived growth factor and hypertrophic stimuli in vascular smooth muscle cells: markers for phenotypic modulation and response to injury. The Journal of biological chemistry. 2005;280:19966-76.

12. Takeji M, Kawada N, Moriyama T, Nagatoya K, Oseto S, Akira S, Hori M, Imai E and Miwa T. CCAAT/Enhancer-binding protein delta contributes to myofibroblast transdifferentiation and renal disease progression. J Am Soc Nephrol. 2004;15:2383-90.

13. Wang B, Chen G, Urabe G, Xie R, Wang Y, Shi X, Guo LW, Gong S and Kent KC. A paradigm of endothelium-protective and stent-free anti-restenotic therapy using biomimetic nanoclusters. Biomaterials. 2018;178:293-301.

14. Marx SO, Totary-Jain H and Marks AR. Vascular smooth muscle cell proliferation in restenosis. Circ Cardiovasc Interv. 2011;4:104-11.

15. Shi Y, Johnson J, Wang B, Chen B, Fisher GL, Urabe G, Shi X, Kent KC, Guo LW and Li L. Mass Spectrometric Imaging Reveals Temporal and Spatial Dynamics of Bioactive Lipids in Arteries Undergoing Restenosis. J Proteome Res. 2019.

16. Ozer HG, El-Gamal D, Powell B, Hing ZA, Blachly JS, Harrington B, Mitchell S, Grieselhuber NR, Williams K, Lai TH, Alinari L, Baiocchi RA, Brinton L, Baskin E, Cannon M, Beaver L, Goettl VM, Lucas DM, Woyach JA, Sampath D, Lehman AM, Yu L, Zhang J, Ma Y, Zhang Y, Spevak W, Shi S, Severson P, Shellooe R, Carias H, Tsang G, Dong K, Ewing T, Marimuthu A, Tantoy C, Walters J, Sanftner L, Rezaei H, Nespi M, Matusow B, Habets G, Ibrahim P, Zhang C, Mathe EA, Bollag G, Byrd JC and Lapalombella R. BRD4 Profiling Identifies Critical Chronic Lymphocytic Leukemia Oncogenic Circuits and Reveals Sensitivity to PLX51107, a Novel Structurally Distinct BET Inhibitor. Cancer Discov. 2018;8:458-477.

17. Zhang MW, B.; Urabe, G.; Ozer, HG.; Han, R.; Kent, KC.; Guo, L-W. Angioplasty-induced epigenomic remodeling entails BRD4 and EZH2 hierarchical regulations. BioRxiv. 2020.

18. Belkina AC, Nikolajczyk BS and Denis GV. BET protein function is required for inflammation: Brd2 genetic disruption and BET inhibitor JQ1 impair mouse macrophage inflammatory responses. J Immunol. 2013;190:3670-8.

19. Gacias M, Gerona-Navarro G, Plotnikov AN, Zhang G, Zeng L, Kaur J, Moy G, Rusinova E, Rodriguez Y, Matikainen B, Vincek A, Joshua J, Casaccia P and Zhou MM. Selective chemical modulation of gene transcription favors oligodendrocyte lineage progression. Chemistry \& biology. 2014;21:841-54.

20. Picaud S, Wells C, Felletar I, Brotherton D, Martin S, Savitsky P, Diez-Dacal B, Philpott M, Bountra C, Lingard H, Fedorov O, Muller S, Brennan PE, Knapp S and Filippakopoulos P. RVX-208, an inhibitor of BET transcriptional regulators with selectivity for the second bromodomain. Proceedings of the National Academy of Sciences of the United States of America. 2013;110:19754-9.

21. Zhao L, Li J, Fu Y, Zhang M, Wang B, Ouellette J, Shahi PK, Pattnaik BR, Watters JJ, Wong WT and Guo LW. Photoreceptor protection via blockade of BET epigenetic readers in a murine model of inherited retinal degeneration. Journal of neuroinflammation. 2017;14:14.

22. Yang ZH, Kitami Y, Takata $Y$, Okura T and Hiwada K. Targeted overexpression of CCAAT/enhancer-binding protein-delta evokes enhanced gene transcription of plateletderived growth factor alpha-receptor in vascular smooth muscle cells. Circulation research. 2001;89:503-8.

23. Kaplan-Albuquerque N, Van Putten V, Weiser-Evans MC and Nemenoff RA. Depletion of serum response factor by RNA interference mimics the mitogenic effects of platelet 
derived growth factor-BB in vascular smooth muscle cells. Circulation research. 2005;97:427-33.

24. Meloche J, Lampron MC, Nadeau V, Maltais M, Potus F, Lambert C, Tremblay E, Vitry G, Breuils-Bonnet S, Boucherat O, Charbonneau E, Provencher S, Paulin R and Bonnet S. Implication of Inflammation and Epigenetic Readers in Coronary Artery Remodeling in Patients With Pulmonary Arterial Hypertension. Arteriosclerosis, thrombosis, and vascular biology. 2017;37:1513-1523.

25. Gomez D, Baylis RA, Durgin BG, Newman AAC, Alencar GF, Mahan S, St Hilaire C, Muller W, Waisman A, Francis SE, Pinteaux E, Randolph GJ, Gram H and Owens GK. Interleukin-1 beta has atheroprotective effects in advanced atherosclerotic lesions of mice. Nature medicine. 2018;24:1418-1429.

26. Takata Y, Kitami Y, Yang ZH, Nakamura M, Okura T and Hiwada K. Vascular inflammation is negatively autoregulated by interaction between CCAAT/enhancer-binding protein-delta and peroxisome proliferator-activated receptor-gamma. Circulation research. 2002;91:427-33.

27. Hogan NT, Whalen MB, Stolze LK, Hadeli NK, Lam MT, Springstead JR, Glass CK and Romanoski CE. Transcriptional networks specifying homeostatic and inflammatory programs of gene expression in human aortic endothelial cells. eLife. 2017;6.

28. Brown JD, Feldman ZB, Doherty SP, Reyes JM, Rahl PB, Lin CY, Sheng Q, Duan Q, Federation AJ, Kung AL, Haldar SM, Young RA, Plutzky J and Bradner JE. BET bromodomain proteins regulate enhancer function during adipogenesis. Proceedings of the National Academy of Sciences of the United States of America. 2018;115:2144-2149.

29. Brown JD, Lin CY, Duan Q, Griffin G, Federation AJ, Paranal RM, Bair S, Newton G, Lichtman AH, Kung AL, Yang T, Wang H, Luscinskas FW, Croce KJ, Bradner JE and Plutzky J. NF-kappaB directs dynamic super enhancer formation in inflammation and atherogenesis. Molecular cell. 2014;56:219-31.

30. Lee JE, Park YK, Park S, Jang Y, Waring N, Dey A, Ozato K, Lai B, Peng W and Ge K. Brd4 binds to active enhancers to control cell identity gene induction in adipogenesis and myogenesis. Nature communications. 2017;8:2217.

31. Zhang Y, Liu T, Meyer CA, Eeckhoute J, Johnson DS, Bernstein BE, Nusbaum C, Myers RM, Brown M, Li W and Liu XS. Model-based analysis of ChIP-Seq (MACS). Genome biology. 2008;9:R137.

32. Zang C, Schones DE, Zeng C, Cui K, Zhao K and Peng W. A clustering approach for identification of enriched domains from histone modification ChIP-Seq data. Bioinformatics. 2009;25:1952-8.

33. Shi X, Guo LW, Seedial S, Takayama T, Wang B, Zhang M, Franco SR, Si Y, Chaudhary MA, Liu B and Kent KC. Local CXCR4 Upregulation in the Injured Arterial Wall Contributes to Intimal Hyperplasia. Stem Cells. 2016;34:2744-2757.

34. Xie X, Urabe G, Marcho L, Stratton M, Guo LW and Kent CK. ALDH1A3 Regulations of Matricellular Proteins Promote Vascular Smooth Muscle Cell Proliferation. iScience. 2019;19:872-882. 


\section{Figure legends}

\section{Figure 1. Injury-induced enrichment of BRD4 and H3K27ac at Cebpd in rat carotid arteries}

Balloon-injured rat left common carotid arteries and contralateral arteries (uninjured control) were collected at day 7 post angioplasty and snap frozen until use for ChIPseq.

A. ChIPseq binding density at Cebod. Shown are ChIPseq peak profiles for BRD4 and histone marks. Data were obtained from injured and uninjured arteries. Non-specific input indicates low background noise.

B. Venn diagrams showing overlap of ChIPseq peaks for H3K27ac and BRD4.

\section{Figure 2. Silencing BRD4 reduces CEBPD mRNA and protein in SMCs}

MOVAS cells were transfected with scrambled or specific siRNA for $24 \mathrm{~h}$, cultured with fresh medium (no RNAi Max) for another 24-48h, and then harvested for qRT-PCR (A) or Western blot (B and $\mathrm{C}$ ) analysis, respectively.

Quantification: Readings from triplicate qRT-PCR reactions were normalized (to GAPDH) and averaged. The average values from 3 independent repeat experiments were then averaged again to calculate mean \pm SEM $(n=3)$. Densitometry of Western blots from independent repeat experiments was normalized (to $\beta$-actin, similar band intensity among blots) and then averaged to calculate mean \pm SEM $(n=3)$.

Statistics: One-way analysis of variance (ANOVA) followed by Bonferroni post-hoc test; * $\mathrm{P}<0.05$, ${ }^{* *} \mathrm{P}<0.01$.

\section{Figure 3. Expression of dominant-negative BD1 of BRD4 inhibits CEBPD expression}

A. Diagram of the constructs used to exogenously express GFP (control) and a dominantnegative domain competing with the BD1 or BD2 of BRD4.

B and C. Effect of exogenously expressed BD1 or BD2 (of BRD4) on CEBPD expression.

D. Diagram to show selective binding of olinone and RVX208 to BD1 and BD2, respectively, and binding of JQ1 to both; each of the bromodomain blockers binds all three BETs (BRD2, BRD3, BRD4).

$\mathbf{E}$ and $\mathbf{F}$. Effect of bromodomain blockers on CEBPD expression. Data from vehicle blots were pooled into a single value bar in the plot.

MOVAS cells were transfected with the dominant-negative BD1 or BD2 plasmid for 24h and cultured in fresh medium (no Lipofectamine) for another 24h before harvest for Western blot analysis. For pharmacological pretreatment, cells were incubated with vehicle (equal amount of DMSO) or a bromodomain blocker for $4 \mathrm{~h}$ before harvest.

Quantification: Densitometry of Western blots from independent repeat experiments was normalized (to $\beta$-actin, similar band intensity among blots) and then averaged to calculate mean $\pm \operatorname{SEM}(n=3)$.

Statistics: One-way analysis of variance (ANOVA) followed by Bonferroni post-hoc test; * $P<0.05$, ${ }^{* *} \mathrm{P}<0.01$. 


\title{
Figure 4. Co-immunoprecipitation of endogenous BRD4 with CEBPD
}

MOVAS cells stably expressing HA-tagged empty vector (EV) or HA-tagged CEBPD were incubated with vehicle (equal amount of DMSO) or bromodomain blocker JQ1 $(1 \mu \mathrm{M})$ or HDAC inhibitor TSA $(1 \mu \mathrm{M})$ for $24 \mathrm{~h}$. The cells were then harvested for IP of CEBPD using an antibody against the HA tag. A. Quantified data. B. Representative Western blots.

Quantification: Densitometry of Western blots from independent repeat experiments was normalized (to $\beta$-actin, similar band intensity among blots) and then averaged to calculate mean $\pm \operatorname{SEM}(n=6)$.

Statistics: One-way analysis of variance (ANOVA) followed by Bonferroni post-hoc test; ${ }^{*}<<0.05$, ${ }^{* *} \mathrm{P}<0.01$.

\section{Figure 5. Chromatin immunoprecipitation of Cebpd promoter DNA with CEBPD or endogenous BRD4}

\author{
A-D. ChIP against HA-tagged CEBPD in SMCs. \\ E-F. ChIP against endogenous BRD4 in SMCs (E) or HEK293 cells (F).
}

MOVAS cells for stable expression of HA-tagged empty vector (EV) or HA-tagged CEBPD (OECEBPD) were starved in basal medium $(0.5 \% \mathrm{FBS})$ for $24 \mathrm{~h}$, pretreated with vehicle or bromodomain blocker JQ1 $(1 \mu \mathrm{M})$ for $2 \mathrm{~h}$, and then treated with TNF $\alpha$ (final $20 \mathrm{ng} / \mathrm{ml}$ ) for $24 \mathrm{~h}$ prior to harvest for ChIP-qPCR analysis.

Quantification: Readings from triplicate qRT-PCR reactions were normalized (to GAPDH) and averaged. The average values from at least 3 independent repeat experiments were then averaged again to calculate mean \pm SEM $(n=3-5)$.

Statistics: One-way analysis of variance (ANOVA) followed by Bonferroni post-hoc test; ${ }^{*}<<0.05$, ${ }^{* *} \mathrm{P}<0.01,{ }^{* * *} \mathrm{P}<0.001$.

\section{Figure 6. Role of CEBPD in the inflammatory SMC state transition}
A and B. CEBPD gain of function. A shows CEBPD overexpression.
$\mathbf{C}$ and $\mathbf{D}$. CEBPD loss of function. $C$ indicates effective CEBPD silencing.
E. Treatment of SMCs with TNF $\alpha$ upregulates BRD4 protein (Movas cell line).
F. Effect of pretreatment with bromodomain blocker JQ1.

MOVAS cells for stable expression of HA-tagged empty vector (EV) or HA-tagged CEBPD (OECEBPD) were starved in basal medium $(0.5 \% \mathrm{FBS})$ for $24 \mathrm{~h}$, pretreated with vehicle or JQ1 (1 $\mu \mathrm{M}$ ) for $2 \mathrm{~h}$, and then treated with TNF $\alpha$ (final $20 \mathrm{ng} / \mathrm{ml}$ ) for $24 \mathrm{~h}$ prior to harvest for qRT-PCR (mRNA) or Western blot (protein) analysis. For CEBPD silencing, MOVAS cells were transfected with siRNA for $24 \mathrm{~h}$ and cultured in fresh starvation medium $(0.5 \% \mathrm{FBS})$ for another 24h prior to TNF $\alpha$ treatment.

Quantification: Readings from triplicate qRT-PCR reactions were normalized (to GAPDH) and averaged. The average values from at least 3 independent repeat experiments were then averaged again to calculate mean \pm SEM $(n=3-5)$. 
Statistics: One-way analysis of variance (ANOVA) followed by Bonferroni post-hoc test; * $\mathrm{P}<0.05$, ${ }^{* *} \mathrm{P}<0.01,{ }^{* * *} \mathrm{P}<0.001$.

\section{Figure 7. Preferential regulation of PDGFR $\alpha$ over PDGFR $\beta$ in SMCs}

A - D. Effect of JQ1 pretreatment on TNF $\alpha$-stimulated upregulation of CEBPD and PDGFR $\alpha$. A shows concentration-dependent effect of JQ1 on reducing CEBPD mRNA levels.

Representative Western blots are shown in C.

$\mathbf{E}$ and $\mathbf{F}$. CEBPD gain of function. Representative Western blots are shown in E.

$\mathbf{G}$ and $\mathbf{H}$. CEBPD loss of function. Representative Western blots are shown in G.

MOVAS cells for stable expression of HA-tagged empty vector (EV) or HA-tagged CEBPD (OECEBPD) were starved in basal medium $(0.5 \% \mathrm{FBS})$ for $24 \mathrm{~h}$, pretreated with vehicle or JQ1 (1 $\mu \mathrm{M}$ ) for $2 \mathrm{~h}$, and then treated with TNF $\alpha$ (final $20 \mathrm{ng} / \mathrm{ml}$ ) for $24 \mathrm{~h}$ prior to harvest for qRT-PCR (mRNA) or Western blot (protein) analysis. For CEBPD silencing, MOVAS cells were transfected with siRNA for $24 \mathrm{~h}$ and cultured in fresh starvation medium $(0.5 \% \mathrm{FBS})$ for another $24 \mathrm{~h}$ prior to TNF $\alpha$ treatment.

Quantification: Readings from triplicate qRT-PCR reactions were normalized (to GAPDH) and averaged. The average values from at least 3 independent repeat experiments were then averaged again to calculate mean \pm SEM $(n=3-5)$. Densitometry of Western blots from independent repeat experiments was normalized (to $\beta$-actin, similar band intensity among blots) and then averaged to calculate mean \pm SEM $(n=4)$.

Statistics: One-way analysis of variance (ANOVA) followed by Bonferroni post-hoc test; * $P<0.05$, ${ }^{* *} \mathrm{P}<0.01,{ }^{* *} \mathrm{P}<0.001$.

\section{Figure 8. Schematic proposal for a BRD4/CEBPD partnership associated with chromatin}

TNF $\alpha$ as extracellular signal stimulates upregulation of epigenetic reader protein BRD4 which, while reading $\mathrm{H} 3 \mathrm{~K} 27 \mathrm{ac}$, functions in the same complex with transcription factor CEBPD (and unknown co-factors, green circle in the back). BRD4 and CEBPD stock up at enhancers and Cebpd promoter to prompt the transcription of Cebpd (and other target genes); as a result, the inflammatory SMC state transition is propelled. 


\section{Figure 1}

A

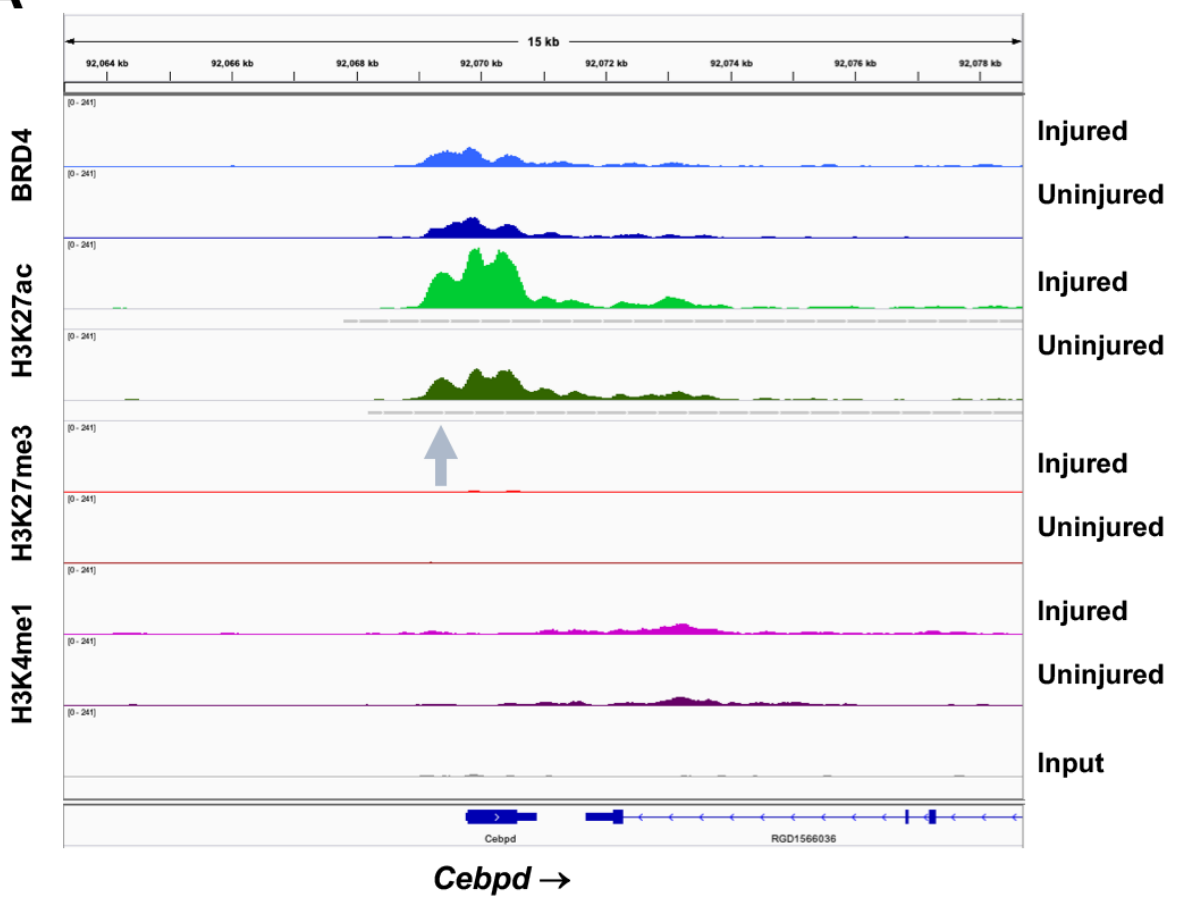

B

Injured

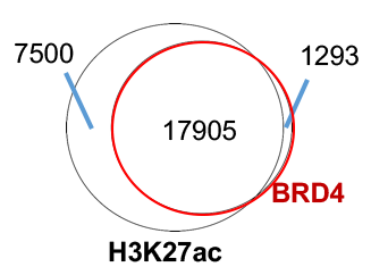

Uninjured

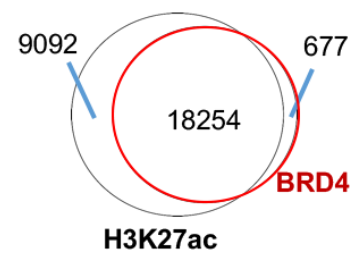


Figure 2

A

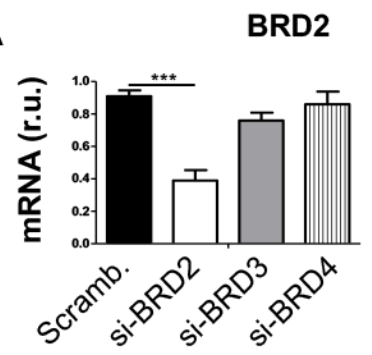

B

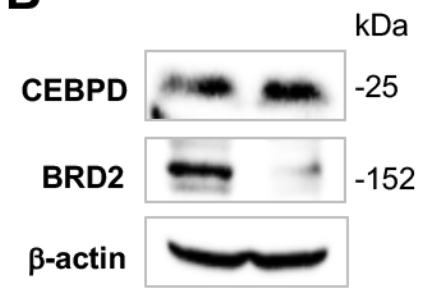

Scrambled si-BRD2

C
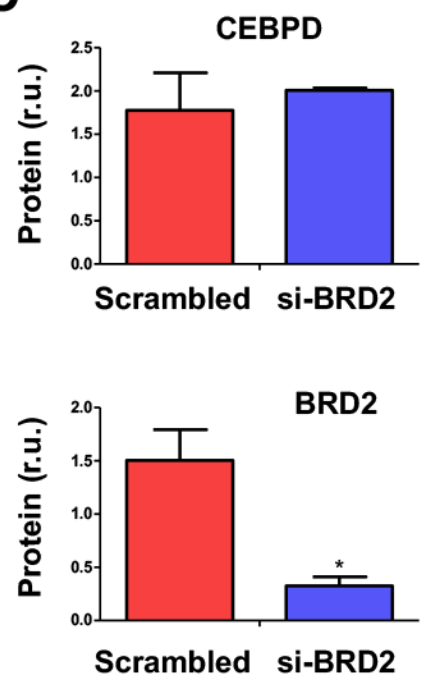

BRD3

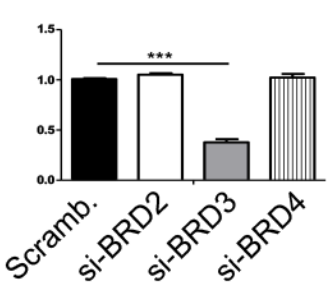

BRD4

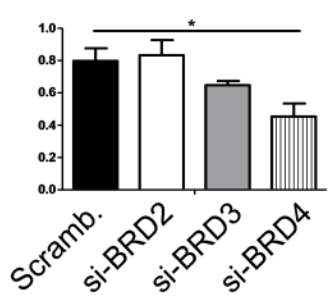

CEBPD

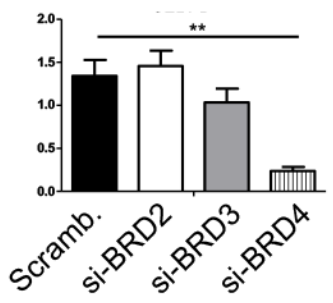

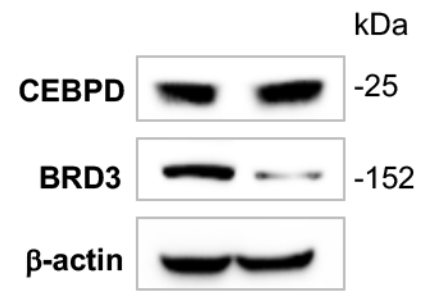

Scrambled si-BRD3
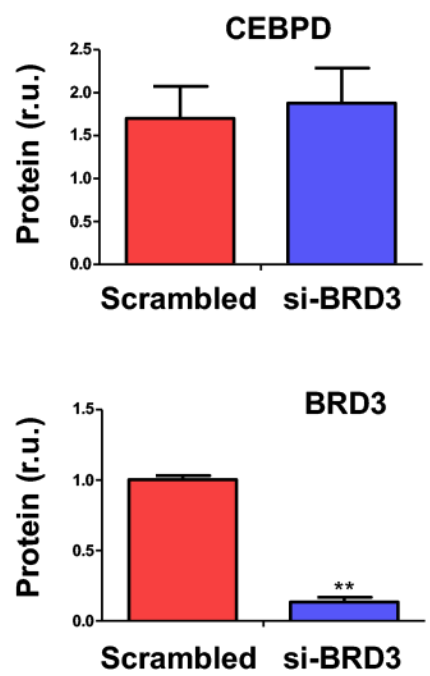

$\mathrm{kDa}$

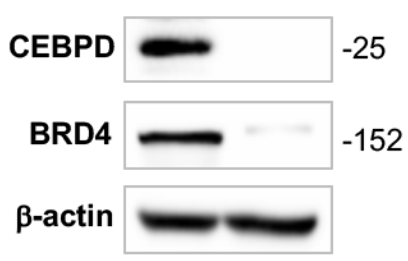

Scrambled si-BRD4
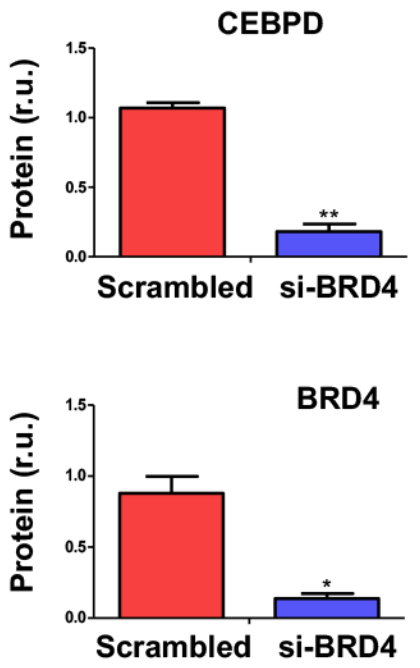
Figure 3

A

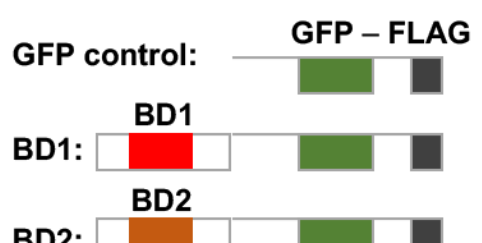

BD2:

D

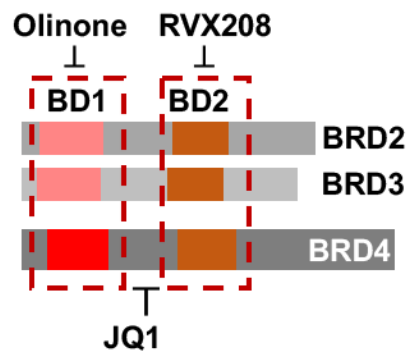

B

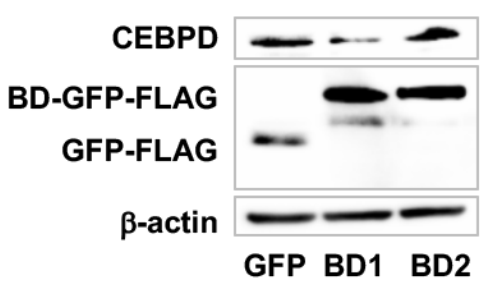

E

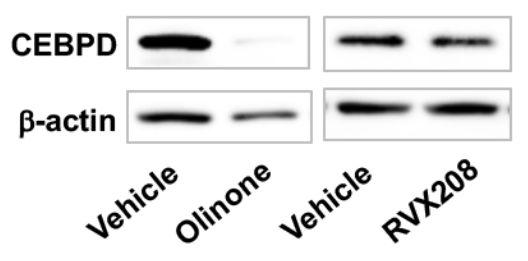

C

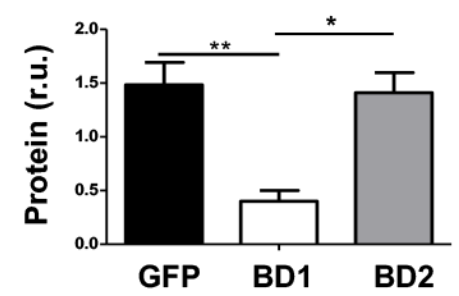

F

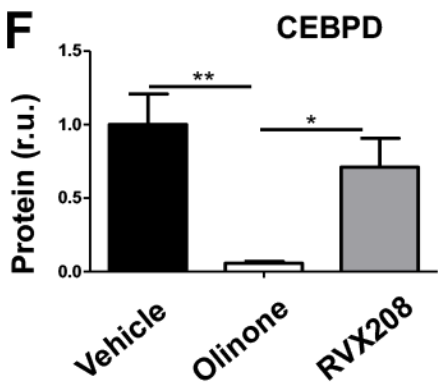


Figure 4
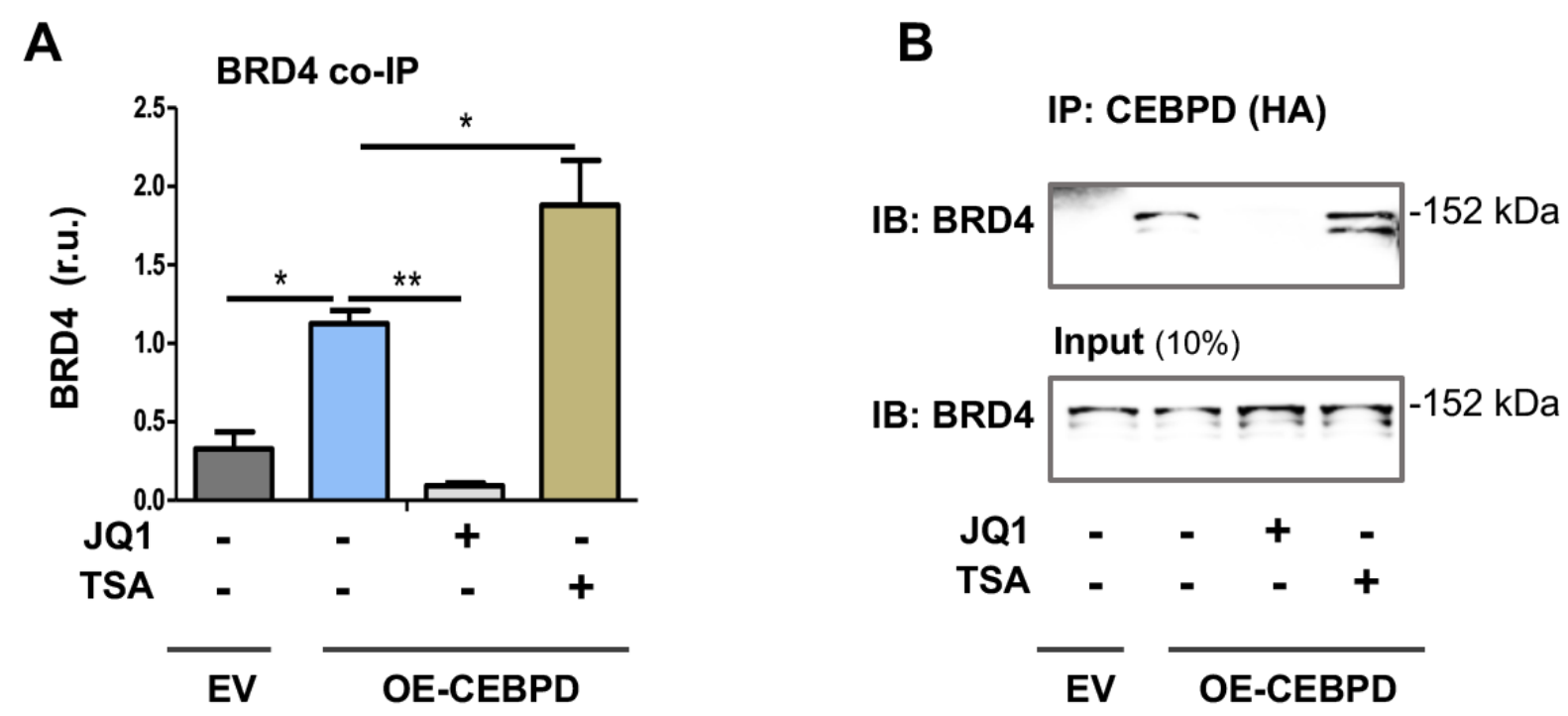
Figure 5

A

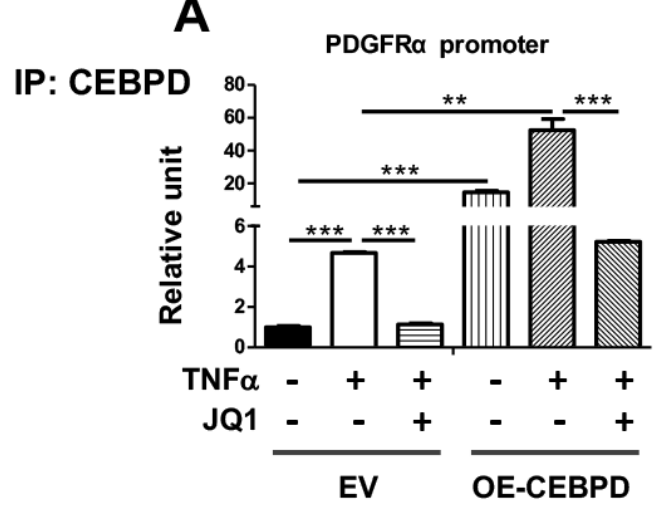

C

IP: CEBPD

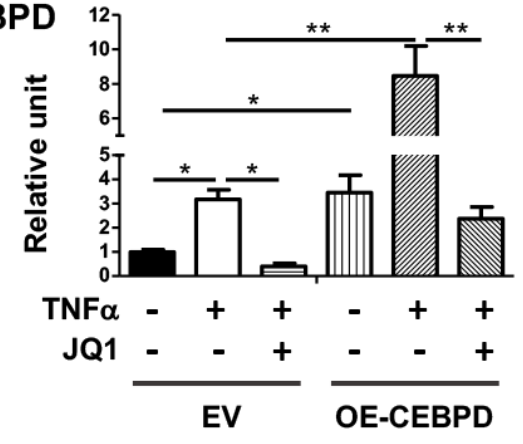

E

IP: BRD4

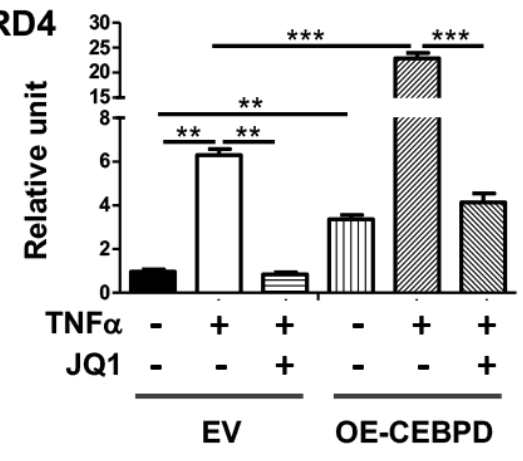

B

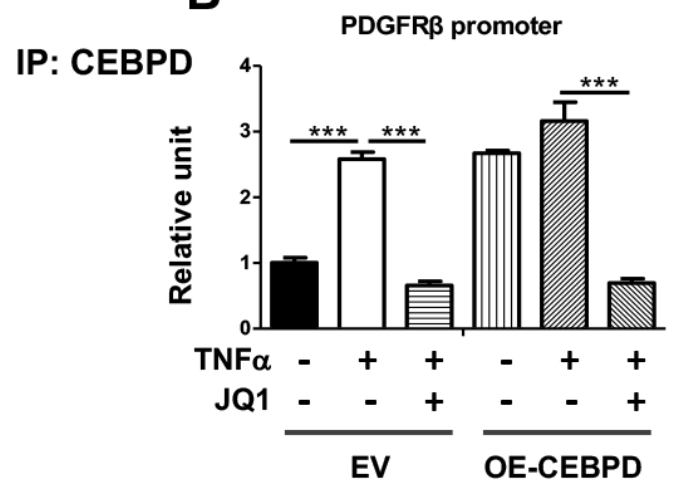

D

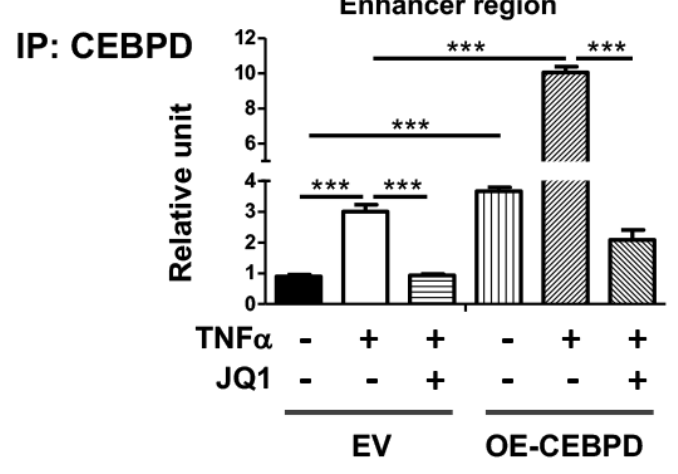

F

CEBPD promoter (HEK293A)

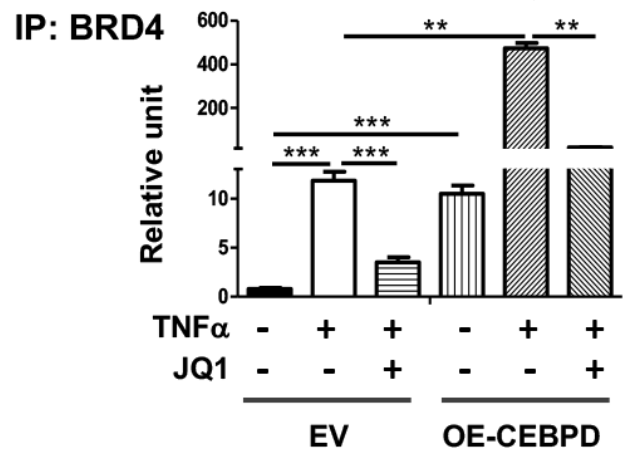


Figure 6

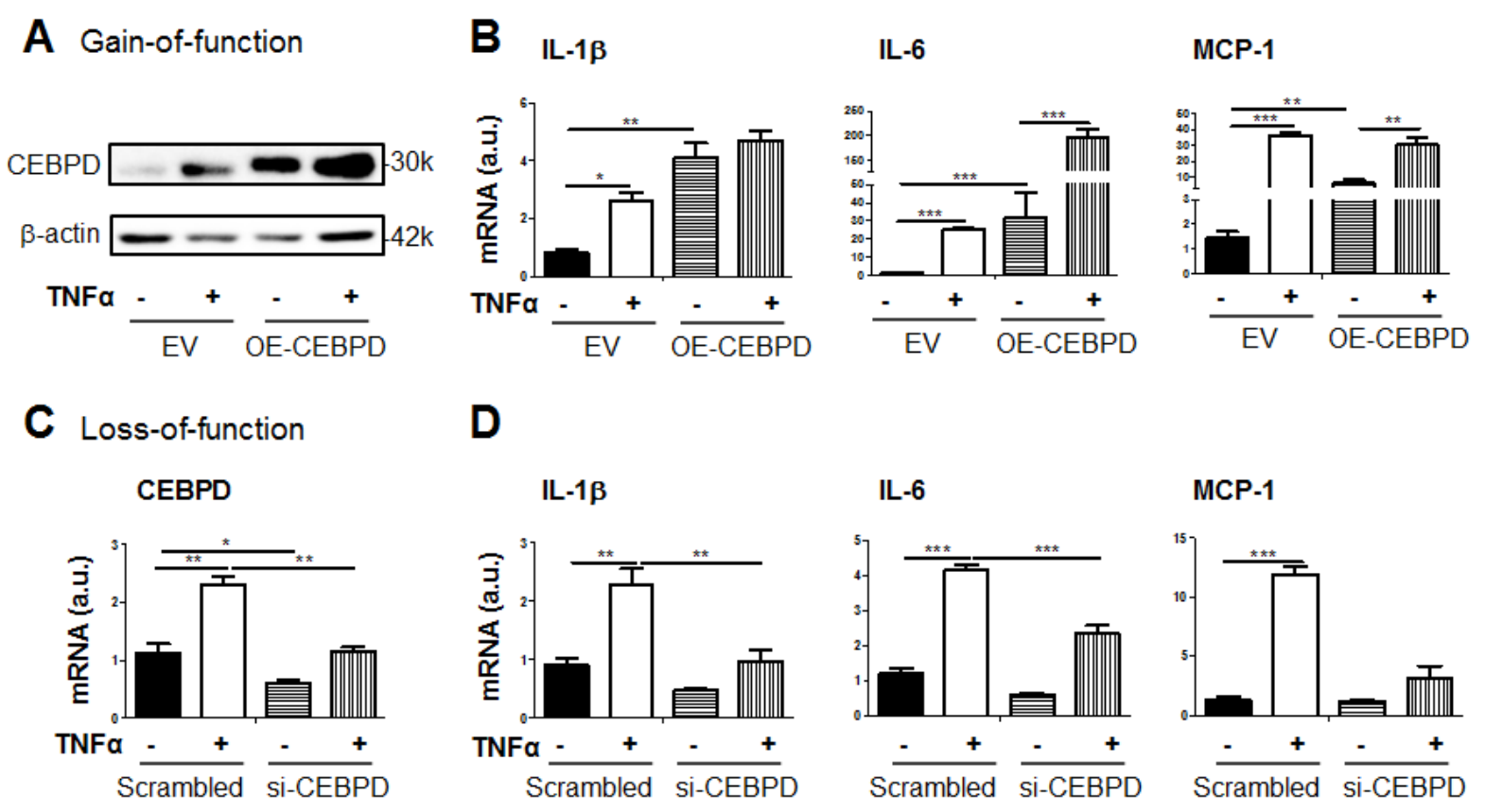

E TNFa's effect on BRD4
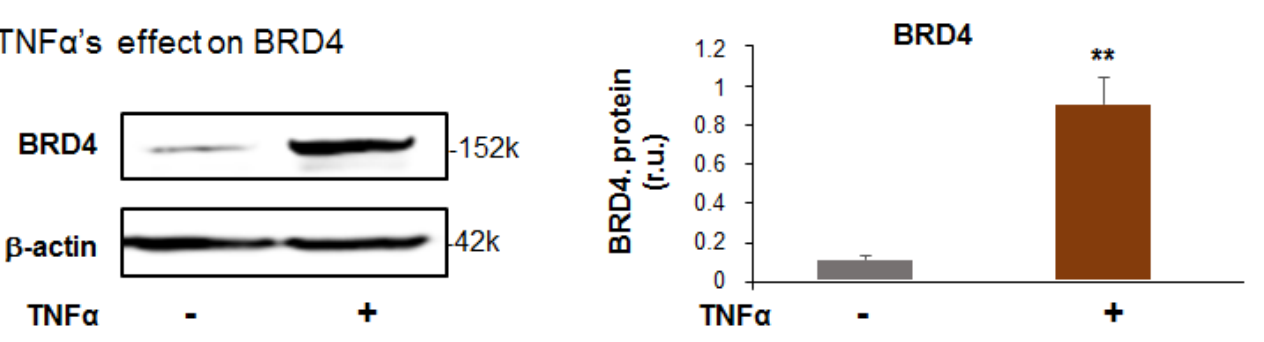

F JQ1 pre-treatment

\section{CEBPD}

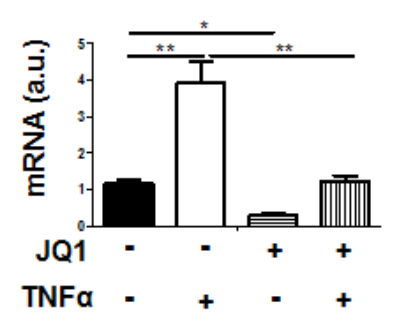

IL-1及

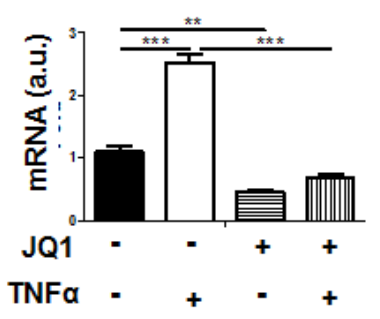

IL-6

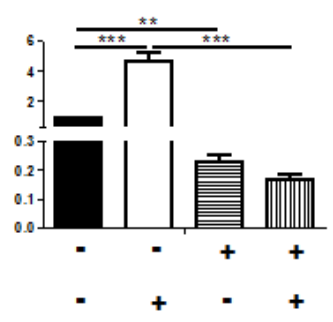

MCP-1

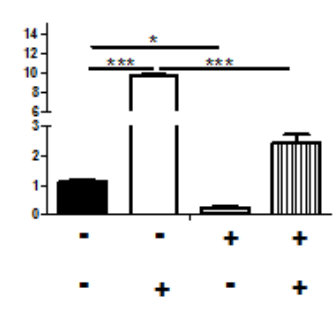


A JQ1 pre-treatment

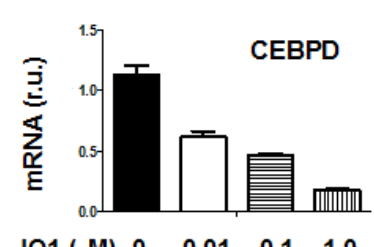

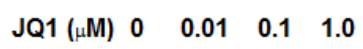

B

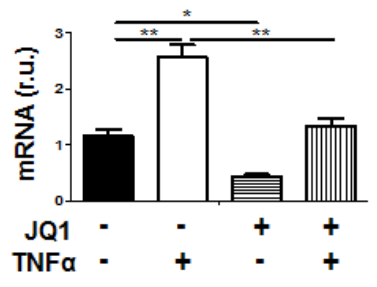

D CEBPD

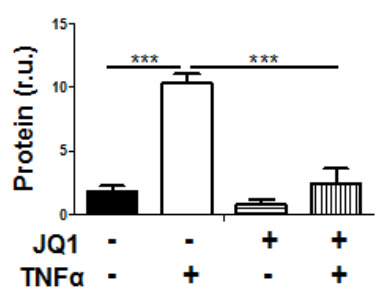

JQ1
TNFa

\section{JQ1 pre-treatment}

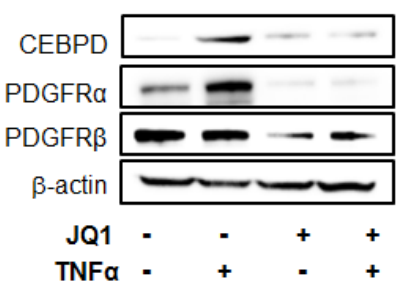

\section{E}
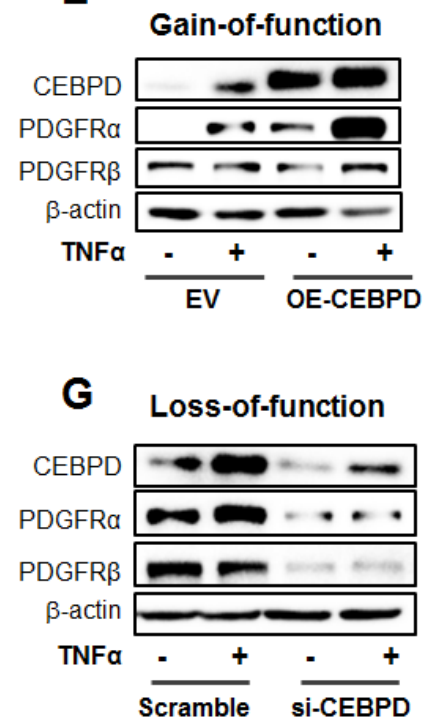

F

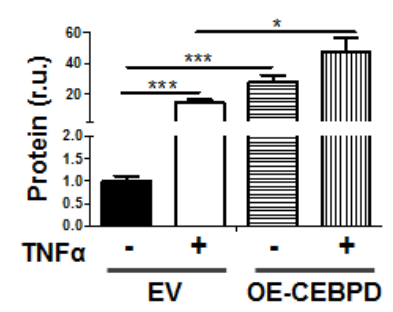

H

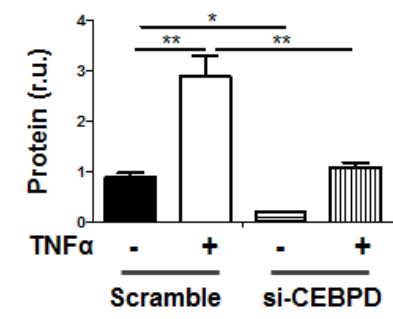

PDGFR $\alpha$

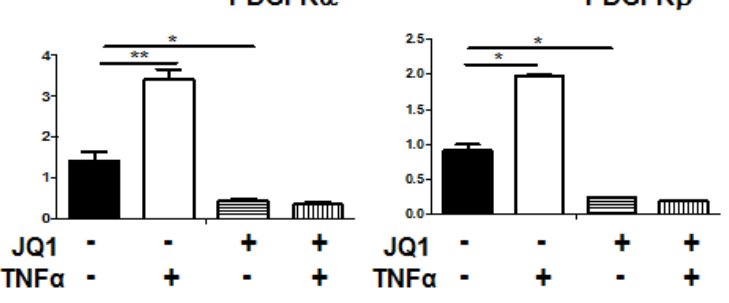

PDGFR $\alpha$

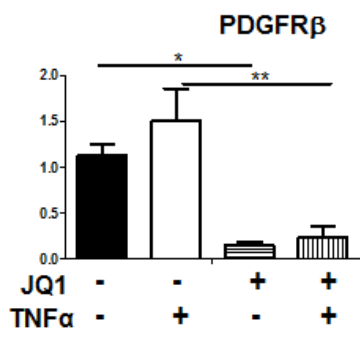

PDGFR $\beta$
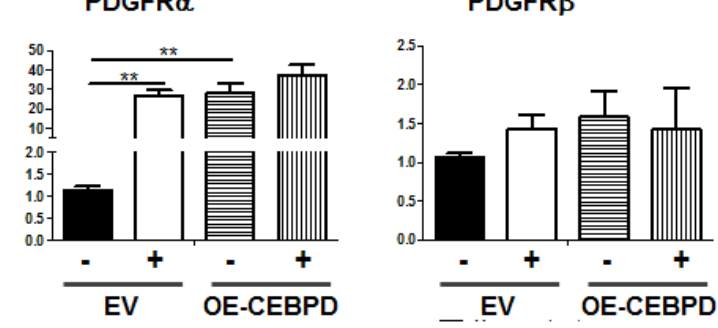
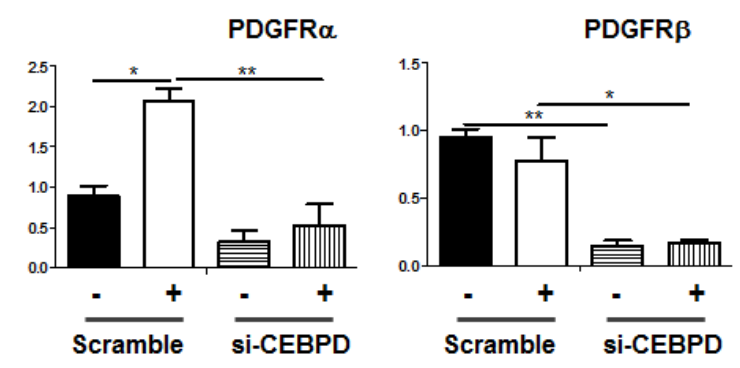
Figure 8

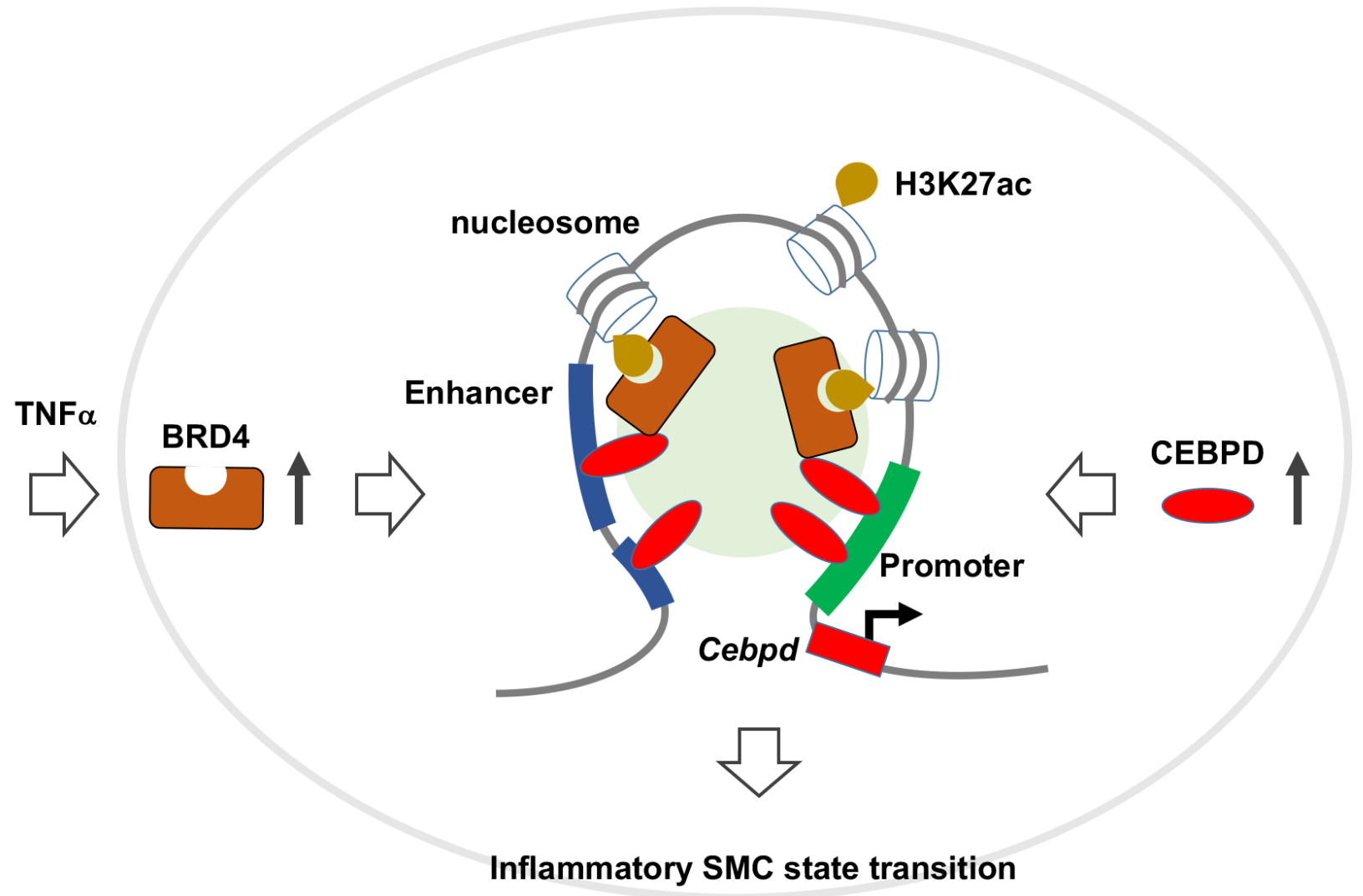




\section{Supplemental Tables}

Table S1. Kits and reagents

\begin{tabular}{|l|l|l|}
\hline Product & Manufacturer & Catalog Number \\
\hline Protein A/G beads & Thermo Scientific & 88802 \\
\hline RNAi Max & Invitrogen & 13778150 \\
\hline Protease inhibitor cocktail & Thermo Scientific & 87785 \\
\hline jetPRIME transfection reagent & VWR & $114-07$ \\
\hline PageRuler TM Plus Prestained Protein & Thermo Scientific & 26620 \\
\hline Ladder & & $170-5060$ \\
\hline QIAGity Western ECL Substrate & Bio-rad & 12143 \\
\hline PureLink ${ }^{\text {TM }}$ Quick Plasmid Miniprep Kit & Invitrogen & K210011 \\
\hline
\end{tabular}

Table S2. Antibodies

\begin{tabular}{|l|l|l|l|l|}
\hline Antigen & Manufacturer & Catalog Number & Dilution Ratio & Application \\
\hline BRD2 & Proteintech & $22236-1-A P$ & $1: 1000$ & Western Blot \\
\hline BRD3 & Proteintech & $11859-1-A P$ & $1: 1000$ & Western Blot \\
\hline BRD4 & Abcam & ab128874 & $1: 1000$ & Western Blot \\
\hline
\end{tabular}




\begin{tabular}{|l|l|l|l|l|}
$\beta$-actin & Proteintech & $66009-1-\mathrm{Ig}$ & $1: 5000$ & Western Blot \\
\hline HA & Proteintech & $51064-2-$ AP & $1: 5000$ & IP \\
\hline
\end{tabular}

Table S3. siRNA or shRNA sequences for mouse and rat genes

\begin{tabular}{|l|l|}
\hline \multirow{2}{*}{ Mouse CEBPD siRNA } & \\
\cline { 2 - 2 } & \\
\hline Rat CEBPD shRNA\#1 & CCGGTCGACTTCAGCGCCTACATTGCTCGAGCAATGTAGGCGCTGAAGTCGATTTTTG \\
\cline { 2 - 2 } Rat CEBPD shRNA\#2 & AATTCAAAAATCGACTCAGCGCCTACATTGCTCGAGCAATGTAGGCGCTGAAGTCGA \\
\hline Mouse BRD2 shRNA & AATTCAAACCACTTCAACAGCAATCTCGAGATGGTGTTGAAGAGGTCGGCTTTTG \\
\cline { 2 - 2 } & \\
\hline \multirow{2}{*}{ Mouse BRD3 shRNA } & \\
\cline { 2 - 2 } Mouse BRD4 shRNA & \\
\cline { 2 - 2 } &
\end{tabular}

Table S4. Primer sequences for mouse genes

\begin{tabular}{|c|c|}
\hline \multirow{2}{*}{ Mouse GAPDH } & Forward: AAGGTCGGTGTGAACGGATTT \\
\hline & Reverse: CTTTGTCACAAGAGAAGGCAGC \\
\hline \multirow{2}{*}{ Mouse BRD2 } & Forward: AATGGCTTCTGTACCAGCTTTAC \\
\hline & Reverse: CTGGCTTTTTGGGATTGGACA \\
\hline \multirow{2}{*}{ Mouse BRD3 } & Forward: AAAAAGGCTCCCACCAAGAAG \\
\hline & Reverse: TGTCAAGGCTAAGTTGTCGCT \\
\hline \multirow{2}{*}{ Mouse BRD4 } & Forward: AGTGTCTTTGACCCTATTAGCCA \\
\hline & Reverse: CATGCTGGTTGAGATGGGG \\
\hline \multirow{2}{*}{ Mouse CEBPD } & Forward: CGACTTCAGCGCCTACATTGA \\
\hline & Reverse: CTAGCGACAGACCCCACAC \\
\hline \multirow{2}{*}{ Mouse PDGFRa } & Forward: TCCATGCTAGACTCAGAAGTCA \\
\hline & Reverse: TCCCGGTGGACACAATTTTTC \\
\hline \multirow{2}{*}{ Mouse IL1-beta } & Forward: GCAACTGTTCCTGAACTCAACT \\
\hline & Reverse: ATCTTTTGGGGTCCGTCAACT \\
\hline \multirow{2}{*}{ Mouse IL-6 } & Forward: CTGCAAGAGACTTCCATCCAG \\
\hline & Reverse: AGTGGTATAGACAGGTCTGTTGG \\
\hline \multirow{2}{*}{ mouse MCP-1 } & Forward: TTAAAAACCTGGATCGGAACCAA \\
\hline & Reverse: GCATTAGCTTCAGATTTACGGGT \\
\hline \multirow{2}{*}{ Mouse PDGFR $\beta$} & Forward: CAAGAAGCGGCCATGAATCAG \\
\hline & Reverse: CGGCCCTAGTGAGTTGTTGT \\
\hline
\end{tabular}

